Yong Zhang*, Kejun Chen, and Wen Li

\title{
Existence of strongly symmetrical weakly pandiagonal graeco-latin squares
}

https://doi.org/10.1515/spma-2018-0013

Received December 6, 2017; accepted March 21, 2018

\begin{abstract}
A graeco-latin sauare is a pair of orthogonal latin squares. It is a design of experiment in which the experimental units are grouped in three different ways. In this paper, constructions of a pair of orthogonal latin sauares which are both strongly symmetrical and weakly pandiagonal are investigated. As a result, it is proved that there exists a pair of strongly symmetrical weakly pandiagonal orthogonal latin sauare of order $n$ if and only if $n>4$ and $n \equiv 0,1,3(\bmod 4)$ with only one possible exception for $n=12$.
\end{abstract}

Keywords: Latin square, graeco, orthogonal, strongly symmetrical, weakly pandiagonal

\section{Introduction}

A Latin square of order $n$, denoted by $\operatorname{LS}(n)$, is an $n \times n$ array such that every row and every column is a permutation of an $n$-set $S$. A transversal in a Latin square is a set of positions, one per row and one per column, among which the symbols occur precisely once each. A diagonal Latin square is a Latin square with the additional property that the main diagonal and back diagonal are both transversals. Two LS $(n)$ s are called orthogonal, denoted by $\operatorname{OLS}(n)$, if each symbol in the first square meets each symbol in the second square exactly once when they are superposed. A pair of orthogonal latin squares is also called a graeco-latin sauare because Euler ([7]) used Greek letters for one square of the pair and latin letters for the other. See also [5] for overview of the Latin squares.

In 1776, Euler presented a paper in which he constructed magic squares of orders 3, 4, and 5 from orthogonal latin squares. He posed the question for order 6, now known as Eulers 36 Officers Problem. He conjectured that no solution exists for order 6. Indeed he conjectured further that there exist orthogonal latin squares of all orders $n$ except when $n \equiv 2(\bmod 4)([7,9])$. Much later, Bose, Shrikhande and Parker [1] proved that the Euler conjecture was false for all orders $n$ of the form $4 k+2$ except $n=2$ or 6 . Much shorter disproofs of Euler's conjecture have been obtained. See, for example, [4, 15].

Wallis and Zhu[11], Heinrich and Hilton[10], Brown, Cherry, Most, Most, Parker and Wallis [2] investigated the existence of orthogonal diagonal latin squares. They proved that there exists a pair of diagonal OLS $(n) \mathrm{s}$ if and only if $n \notin\{2,3,6\}$.

$\operatorname{An} \operatorname{LS}(n)$ is called self-orthogonal if it is orthogonal to its transpose. Let $n$ be a positive integer. Denote $I_{n}=\{0,1, \cdots, n-1\}$. An LS $(n)$ over $I_{n} A=\left(a_{i, j}\right)\left(i, j \in I_{n}\right)$ is called strongly symmetrical if $a_{i, j}+a_{n-1-i, n-1-j}=$ $n-1, i, j \in I_{n}$. Danhof, Phillips and Wallis [6] investigated the existence of strongly symmetrical OLS(n)s. $\mathrm{Du}$ and Cao[8] investigated the existence of strongly symmetrical self-orthogonal $\mathrm{LS}(n)$. Cao and Li [3] proved that there is a strongly symmetrical self-orthogonal $\operatorname{LS}(n)$ if and only if $n \equiv 0,1,3(\bmod 4)$ and $n \neq 3$. It results in the following.

\footnotetext{
${ }^{\star}$ Corresponding Author: Yong Zhang: School of Mathematics and Statistics, Yancheng Teachers University, Jiangsu 224002, P. R. China, E-mail: zyyctc@126.com

Kejun Chen: School of Mathematics and Information Science, Nanjing Normal University of Special Education, Nanjing, Jiangsu, 210038, P. R. China

Wen Li: School of Science, Xichang University, Sichuan 615013, P. R. China
} 
Lemma 1.1. ([3]) A pair of strongly symmetrical OLS(n) exists if and only if $n \equiv 0,1,3(\bmod 4)$ and $n \neq 3$.

Given positive integers $a, n$, we denote $a(\bmod n)$ by $\langle a\rangle_{n}$ for convenience. For a matrix $A$ of order $n$ and $k \in$ $I_{n}$, we call the elements $a_{i,\langle k+i\rangle_{n}}, i \in I_{n}$ the $k$-th right pandiagonal and $a_{i,\langle k-i\rangle_{n}}, i \in I_{n}$ the $k$-th left pandiagonal. Clearly, 0-th right pandiagonal is the main diagonal and the $(n-1)$-th left pandiagonal is the back diagonal. Let $A$ be an $\operatorname{LS}(n)$ over $I_{n}$. A is weakly pandiagonal if the sum of the $n$ numbers in each pandiagonal is the same. For example, the following is a pair of weakly pandiagonal OLS(8).

$$
D=\left(\begin{array}{llllllll}
0 & 3 & 6 & 5 & 4 & 7 & 2 & 1 \\
1 & 2 & 7 & 4 & 5 & 6 & 3 & 0 \\
5 & 6 & 3 & 0 & 1 & 2 & 7 & 4 \\
4 & 7 & 2 & 1 & 0 & 3 & 6 & 5 \\
2 & 1 & 4 & 7 & 6 & 5 & 0 & 3 \\
3 & 0 & 5 & 6 & 7 & 4 & 1 & 2 \\
7 & 4 & 1 & 2 & 3 & 0 & 5 & 6 \\
6 & 5 & 0 & 3 & 2 & 1 & 4 & 7
\end{array}\right), \quad D^{\prime}=\left(\begin{array}{llllllll}
0 & 1 & 5 & 4 & 2 & 3 & 7 & 6 \\
3 & 2 & 6 & 7 & 1 & 0 & 4 & 5 \\
6 & 7 & 3 & 2 & 4 & 5 & 1 & 0 \\
5 & 4 & 0 & 1 & 7 & 6 & 2 & 3 \\
4 & 5 & 1 & 0 & 6 & 7 & 3 & 2 \\
7 & 6 & 2 & 3 & 5 & 4 & 0 & 1 \\
2 & 3 & 7 & 6 & 0 & 1 & 5 & 4 \\
1 & 0 & 4 & 5 & 3 & 2 & 6 & 7
\end{array}\right)
$$

$\mathrm{Xu}$ and $\mathrm{Lu}$ [12] introduced weakly pandiagonal $\operatorname{OLS}(n)$ to construct pandiagonal magic squares. They proved that a weakly pandiagonal self-orthogonal $\operatorname{LS}(n)$ exists if and only if $n \equiv 0,1,3(\bmod 4)$ and $n \neq \equiv 3,6$ $(\bmod 9)$.

Zhang, Li and Lei [13] investigated a pair of weakly pandiagonal OLS $(n)$. They proved the following.

Lemma 1.2. ([13]) A pair of weakly pandiagonal OLS(n) exists if and only if $n \equiv 0,1,3(\bmod 4)$ and $n \neq 3$.

We use SPOLS $(n)$ to denote strongly symmetrical weakly pandiagonal OLS $(n)$. Clearly, the above $\left(D, D^{\prime}\right)$ is a pair of SPOLS(8). In this paper, the existence of a pair of SPOLS $(n)$ is investigated. The construction of $\operatorname{SPOLS}(n)$ is provided in Section 2 and in section 3 the following is proved.

Theorem 1.3. A pair of SPOLS(n) exists if and only if $n>4$ and $n \equiv 0,1,3(\bmod 4)$ with only one possible exception for $n=12$.

\section{Constructions for SPOLS(n)}

Given an $m \times n$ matrix $A$ and an $r \times s$ matrix $B$, the Kronecker Product $A \otimes B$ is an $m r \times n s$ matrix given as follows.

$$
A \otimes B=\left(\begin{array}{cccc}
a_{0,0} B & a_{0,1} B & \cdots & a_{0, n-1} B \\
a_{1,0} B & a_{1,1} B & \cdots & a_{1, n-1} B \\
\vdots & \vdots & & \vdots \\
a_{m-1,0} B & a_{m-1,1} B & \cdots & a_{m-1, n-1} B
\end{array}\right)
$$

Denote $J_{m \times n}$ an $m \times n$ matrix with all elements 1s. A $J_{n \times n}$ is denoted by $J_{n}$. The Kronecker Product is used to get the following construction.

Construction 2.1. If there exists a pair of SPOLS $(m)$ and there exists a pair of SPOLS( $n)$, then there exists a pair of SPOLS(mn).

Proof Suppose that $(A, B)$ is a pair of $\operatorname{SPOLS}(m)$ over $I_{m}$ and $(C, D)$ is a pair of $\operatorname{SPOLS}(n)$ over $I_{n}$. Let

$$
E=\left(e_{i, j}\right)=n A \otimes J_{n}+J_{m} \otimes C, F=\left(f_{i, j}\right)=n B \otimes J_{n}+J_{m} \otimes D,
$$

where

$$
\begin{gathered}
e_{i, j}=n a_{u, s}+c_{v, t}, f_{i, j}=n b_{u, s}+d_{v, t}, \\
i=n u+v, j=n s+t, u, s \in I_{m}, v, t \in I_{n} .
\end{gathered}
$$


In [13] Construction 2.1 proved that $(E, F)$ is a pair of weakly pandiagonal OLS $(m n)$. We shall prove that $E, F$ are also strongly symmetrical.

Let $i^{\prime}=m n-1-i, j^{\prime}=m n-1-j, u^{\prime}=m-1-u, v^{\prime}=n-1-u, s^{\prime}=m-1-s, t^{\prime}=n-1-t$. Clearly, $i^{\prime}=n u^{\prime}+v^{\prime}, j^{\prime}=n s^{\prime}+t^{\prime}$. So we have

$$
\begin{aligned}
e_{i, j}+e_{i^{\prime}, j^{\prime}} & =n a_{u, s}+c_{v, t}+n a_{u^{\prime}, s^{\prime}}+c_{v^{\prime}, t^{\prime}} \\
& =n\left(a_{u, s}+a_{u^{\prime}, s^{\prime}}\right)+\left(c_{v, t}+c_{v^{\prime}, t^{\prime}}\right) \\
& =n(m-1)+n-1=n m-1 .
\end{aligned}
$$

Thus $E$ is strongly symmetrical. Similarly, $F$ is also strongly symmetrical. So $(E, F)$ is a pair of $\operatorname{SPOLS}(m n)$.

Let $m$ be an even integer and $n$ be an integer. Let $T=\left\{T_{0}, T_{1}, \cdots, T_{m-1}\right\}$ be a partition of $I_{m n}$ such that $\left|T_{i}\right|=n, i \in I_{m}$. Denote $S=\frac{m n(m n-1)}{2}$. Given a rational number $x$, we use $\lfloor x\rfloor$ to denote the largest integer $a$ such that $a \leq x$ as usual.

Construction 2.2. Let $\left(D, D^{\prime}\right)$ be a pair of strongly symmetrical OLS $(m)$ over $I_{m}$, and $\left(M, M^{\prime}\right)$ be a pair of strongly symmetrical OLS( $n)$ over $I_{n}$, where $D=\left(d_{u, v}\right), D^{\prime}=\left(d_{u, v}^{\prime}\right), M=\left(m_{i, j}\right), M^{\prime}=\left(m_{i, j}^{\prime}\right)$. Let $\varphi_{u, v}, \varphi_{u, v}^{\prime}$ be a bijection from $I_{n}$ to $T_{v}, u, v \in I_{m}$. Let

$$
\begin{array}{lll}
A=\left(A_{u, v}\right), & A_{u, v}=\left(a_{u, v}(i, j)\right)_{n \times n}, & a_{u, v}(i, j)=\varphi_{u, d_{u, v}}\left(m_{i, j}\right), \\
B=\left(B_{u, v}\right), & B_{u, v}=\left(b_{u, v}(i, j)\right)_{n \times n}, & b_{u, v}(i, j)=\varphi_{u, d_{u, v}^{\prime}}^{\prime}\left(m_{i, j}^{\prime}\right),
\end{array}
$$

where $u, v \in I_{m}, i, j \in I_{n}$. If $\varphi_{u, v}, \varphi_{u, v}^{\prime}$ satisfy

$$
\begin{gathered}
\varphi_{u, v}(t)+\varphi_{m-1-u, m-1-v}(n-1-t)=m n-1, \\
\varphi_{u, v}^{\prime}(t)+\varphi_{m-1-u, m-1-v}^{\prime}(n-1-t)=m n-1,
\end{gathered}
$$

Then $(A, B)$ is a pair of strongly symmetrical OLS $(m n)$ over $I_{m n}$. Further, if $\varphi_{u, v}$ satisfies

$$
\begin{aligned}
& \sum_{u \in I_{m}} \sum_{i \in I_{n}} \varphi_{u, d_{u,\left\langle v+u+\left\lfloor\frac{j+i}{n}\right\rfloor\right\rangle_{m}}}\left(m_{i,\langle j+i\rangle_{n}}\right)=S,
\end{aligned}
$$

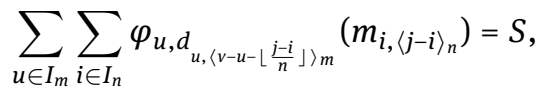

and $\varphi_{u, v}^{\prime}$ satisfies

$$
\begin{aligned}
& \sum_{u \in I_{m}} \sum_{i \in I_{n}} \varphi_{\left.u, d_{u,\langle v+u+}^{\prime}\left[\frac{j+i}{n}\right\rfloor\right\rangle_{m}}^{\prime}\left(m_{i,\langle j+i\rangle_{n}}^{\prime}\right)=S,
\end{aligned}
$$

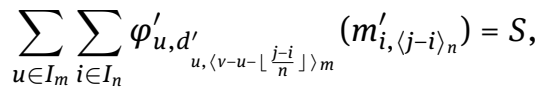

then $(A, B)$ is a pair of SPOLS $(m n)$ over $I_{m n}$.

Proof First we prove that $A, B$ are $\operatorname{LS}(m n)$. Let $u \in I_{m}, i \in I_{n}$. We consider the entries $a_{u, v}(i, j)$ and $a_{u, v^{\prime}}\left(i, j^{\prime}\right)$ of the $(n u+i)$-th row of $A$, where $v, v^{\prime} \in I_{m}, j, j^{\prime} \in I_{n}$, and $(v, j) \neq\left(v^{\prime}, j^{\prime}\right)$. Since $D$ is a latin square, if $v \neq v^{\prime}$ then $d_{u, v} \neq d_{u, v^{\prime}}$, hence $T_{d_{u, v}} \cap T_{d_{u, v^{\prime}}}=\emptyset$. So, $\varphi_{u, d_{u, v}}\left(m_{i, j}\right) \neq \varphi_{u, d_{u, v^{\prime}}}\left(m_{i, j^{\prime}}\right)$. Thus $a_{u, v}(i, j) \neq a_{u, v^{\prime}}\left(i, j^{\prime}\right)$. If $v=v^{\prime}$, $j \neq j^{\prime}$, then the image of $\varphi_{u, d_{u, v}}$ and $\varphi_{u, d_{u, v^{\prime}}}$ fall into the same set $T_{d_{u, v}}$. Since $M$ is latin square, $m_{i, j} \neq m_{i, j^{\prime}}$, thus $\varphi_{u, d_{u, v}}\left(m_{i, j}\right) \neq \varphi_{u, d_{u, v^{\prime}}}\left(m_{i, j^{\prime}}\right)$. So, $a_{u, v}(i, j) \neq a_{u, v^{\prime}}\left(i, j^{\prime}\right)$. Therefore each row of $A$ is a permutation of $I_{m n}$. Similarly, each column of $A$ is also a permutation of $I_{m n}$. Thus $A$ is an $\operatorname{LS}(m n)$ over $I_{m n}$. Similarly, $B$ is also an LS $(m n)$ over $I_{m n}$.

Now we shall prove that $A, B$ are orthogonal. Let

$$
a_{u_{1}, v_{1}}\left(i_{1}, j_{1}\right) b_{u_{1}, v_{1}}\left(i_{1}, j_{1}\right)=a_{u_{2}, v_{2}}\left(i_{2}, j_{2}\right) a_{u_{2}, v_{2}}\left(i_{2}, j_{2}\right)
$$

where $u_{1}, u_{2}, v_{1}, v_{2} \in I_{m}, i_{1}, i_{2}, j_{1}, j_{2} \in I_{n}$. Then 


$$
\begin{aligned}
\varphi_{u_{1}, d_{u_{1}, v_{1}}} & \left(m_{i_{1}, j_{1}}\right)=\varphi_{u_{2}, d_{u_{2}, v_{2}}}\left(m_{i_{2}, j_{2}}\right), \\
\varphi_{u_{1}, d_{u_{1}, v_{1}}^{\prime}}^{\prime}\left(m_{i_{1}, j_{1}}^{\prime}\right) & \left.=\varphi_{u_{2}, d_{u_{2}, v_{2}}^{\prime}}^{\prime}\left(m_{i_{2}, j_{2}}^{\prime}\right)\right) .
\end{aligned}
$$

Note that $\varphi_{u_{i}, d_{u_{i}, v_{i}}}$ and $\varphi_{u_{i}, d_{u_{i}, v_{i}}^{\prime}}^{\prime}$ are bijection from $I_{n}$ to $T_{d_{u_{i}, v_{i}}}$ and $T_{d_{u_{i}, v_{i}}^{\prime}}$, respectively, $i=1$, 2. So, $T_{d_{u_{1}, v_{1}}}=$ $T_{d_{u_{2}, v_{2}}}, T_{d_{u_{1}, v_{1}}^{\prime}}^{\prime}=T_{d_{u_{2}, v_{2}}^{\prime}}$. Thus $d_{u_{1}, v_{1}}=d_{u_{2}, v_{2}}, d_{u_{1}, v_{1}}^{\prime}=d_{u_{2}, v_{2}}^{\prime}$. Since $D, D^{\prime}$ are orthogonal, $u_{1}=u_{2}, v_{1}=v_{2}$. Since $\varphi, \varphi^{\prime}$ are bijection, we have

$$
m_{i_{1}, j_{1}}=m_{i_{2}, j_{2}}, m_{i_{1}, j_{1}}^{\prime}=m_{i_{2}, j_{2}}^{\prime} .
$$

Since $M, M^{\prime}$ are orthogonal, we have $i_{1}=i_{2}, j_{1}=j_{2}$. Thus $A, B$ are orthogonal.

For any $u, v \in I_{m}, i, j \in I_{n}$, let $u^{\prime}=m-1-u, v^{\prime}=m-1-v, i^{\prime}=n-1-i, j^{\prime}=n-1-j$. Since $D, M$ are strongly symmetrical, we get $d_{u, v}+d_{u^{\prime}, v^{\prime}}=m-1, m_{i, j}+m_{i^{\prime}, j^{\prime}}=n-1$. By the condition (L1) we have

$$
\begin{aligned}
a_{u, v}\left(m_{i, j}\right)+a_{u^{\prime}, v^{\prime}}\left(m_{i^{\prime}, j^{\prime}}\right) & =\varphi_{u, d_{u, v}}\left(m_{i, j}\right)+\varphi_{u^{\prime}, d_{u^{\prime}, v^{\prime}}}\left(m_{i^{\prime}, j^{\prime}}\right) \\
& =\varphi_{u, d_{u, v}}\left(m_{i, j}\right)+\varphi_{u^{\prime}, m-1-d_{u, v}}\left(n-1-m_{i, j}\right) \\
& =m n-1 .
\end{aligned}
$$

Thus $A$ is strongly symmetrical. Similar, $B$ is also strongly symmetrical by the condition (L2).

Now we consider the pandiagonals of $A, B$. For any $s \in I_{m n}$, the $s$-th right pandiagonal of $A$ consists of the $\left(k,\langle k+s\rangle_{m n}\right)$-entry of $A, k \in I_{m n}$. Let $k=u n+i, s=v n+j$, where $u, v \in I_{m}, i, j \in I_{n}$. Note that $i+j=n\left\lfloor\frac{i+j}{n}\right\rfloor+\langle i+j\rangle_{n}$, we have

$$
\begin{aligned}
\langle k+s\rangle_{m n}=\langle & n(u+v)+i+j\rangle_{m n} \\
& =\left\langle n\langle u+v\rangle_{m}+i+j\right\rangle_{m n} \\
& =\left\langle n\langle u+v\rangle_{m}+n\left\lfloor\frac{i+j}{n}\right\rfloor+\langle i+j\rangle_{n}\right\rangle_{m n} \\
& =n\left\langle u+v+\left\lfloor\frac{i+j}{n}\right\rfloor\right\rangle_{m}+\langle i+j\rangle_{n} .
\end{aligned}
$$

So, the $\left(k,\langle k+s\rangle_{m n}\right)$-entry of $A$ is

$$
a_{u,\left\langle v+u+\left\lfloor\frac{j+i}{n}\right\rfloor\right\rangle_{m}}\left(i,\langle j+i\rangle_{n}\right)
$$

which is just

$$
\varphi_{\left.\left.u, d_{u,\langle v+u+} \frac{j+i}{n}\right\rfloor\right\rangle_{m}}\left(m_{i,\langle j+i\rangle_{n}}\right) .
$$

The sum of each right pandiagonal of $A$ is the same number $S$ by (L3). Similarly, the sum of each left pandiagonal of $A$ is also $S$ by (L4). So $A$ is weakly pandiagonal. By (L5) and (L6) $B$ is also weakly pandiagonal. Thus $(A, B)$ is a pair of SPOLS $(m n)$ over $I_{m n}$.

Lemma 2.3. There exists a pair of SPOLS(24).

Proof Let $\left(D, D^{\prime}\right)$ be the pair of strongly symmetrical OLS(8) given in the introduction. Let

$$
M=\left(\begin{array}{lll}
0 & 2 & 1 \\
2 & 1 & 0 \\
1 & 0 & 2
\end{array}\right), \quad M^{\prime}=\left(\begin{array}{lll}
1 & 2 & 0 \\
0 & 1 & 2 \\
2 & 0 & 1
\end{array}\right)
$$

Clearly, $\left(M, M^{\prime}\right)$ is a pair of strongly symmetrical OLS(3). Let

$$
\begin{aligned}
& L=\left(\begin{array}{llllllllllll|lll|lll|lll|ll}
0 & 2 & 6 & 3 & 4 & 1 & 8 & 7 & 10 & 11 & 9 & 5 & 21 & 17 & 23 & 20 & 19 & 22 & 15 & 16 & 13 \mid 14 & 18 & 12
\end{array}\right),
\end{aligned}
$$

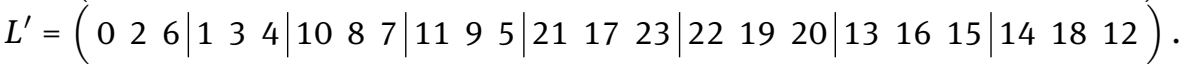

Denote $L=\left(\begin{array}{llll}L_{0} & L_{1} & \cdots & L_{7}\end{array}\right), L_{v}=\left(\begin{array}{ll}l_{v}(0) & l_{v}(1)\end{array} l_{v}(2)\right), v \in I_{8}$. Similarly, we denote $L^{\prime}$.

For any $t \in I_{3}, v \in I_{8}$, let

$$
\varphi_{u, v}(t)=\left\{\begin{array}{lr}
l_{v}(t), & u=0,1,2,3 \\
8 n-1-l_{7-v}(n-1-t), & u=4,5,6,7
\end{array}\right.
$$




$$
\varphi_{u, v}^{\prime}(t)=\left\{\begin{array}{lr}
l_{v}^{\prime}(t), & u=0,1,2,3 \\
8 n-1-l_{7-v}^{\prime}(n-1-t), & u=4,5,6,7
\end{array}\right.
$$

It is readily verified that $\varphi, \varphi^{\prime}$ satisfy (L1)-(L6) in Construction 2.2. Let

$$
\begin{array}{lll}
A=\left(A_{u, v}\right), & A_{u, v}=\left(a_{u, v}(i, j)\right)_{n \times n}, & a_{u, v}(i, j)=\varphi_{u, d_{u, v}}\left(m_{i, j}\right), \\
B=\left(B_{u, v}\right), & B_{u, v}=\left(b_{u, v}(i, j)\right)_{n \times n}, & b_{u, v}(i, j)=\varphi_{u, d_{u, v}^{\prime}}^{\prime}\left(m_{i, j}^{\prime}\right),
\end{array}
$$

where $u, v \in I_{8}, i, j \in I_{3}$. Thus we get a pair of $\operatorname{SPOLS}(24)$ as Construction 2.2, $(A, B)$ as follows.

$$
\begin{aligned}
& \left(\begin{array}{ccc|ccc|ccc|c|c|c|ccc|c|c|c|ccc|ccc}
0 & 6 & 2 & 11 & 5 & 9 & 15 & 13 & 16 & 20 & 22 & 19 & 21 & 23 & 17 & 14 & 12 & 18 & 8 & 10 & 7 & 3 & 1 & 4 \\
6 & 2 & 0 & 5 & 9 & 11 & 13 & 16 & 15 & 22 & 19 & 20 & 23 & 17 & 21 & 12 & 18 & 14 & 10 & 7 & 8 & 1 & 4 & 3
\end{array}\right)
\end{aligned}
$$

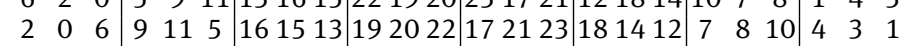

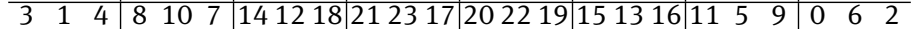

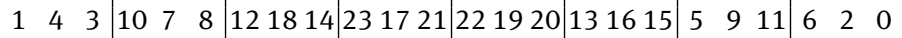

$$
\begin{aligned}
& \begin{array}{lll|lll|lll|lll|lll|lll|lll|lll}
4 & 3 & 1 & 7 & 8 & 10 & 18 & 14 & 12 & 17 & 21 & 23 & 19 & 20 & 22 & 16 & 15 & 13 & 9 & 11 & 5 & 2 & 0 & 6
\end{array}
\end{aligned}
$$

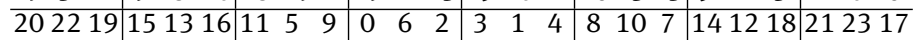

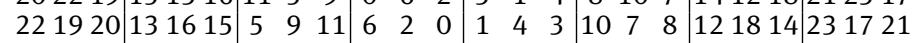

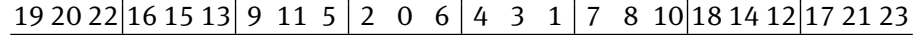

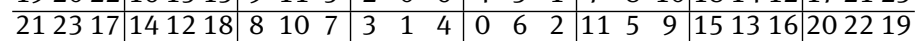

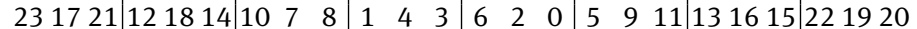

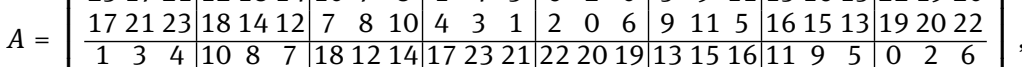

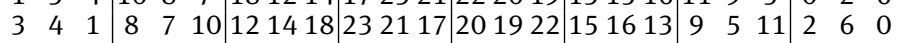

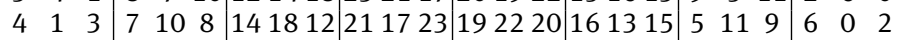

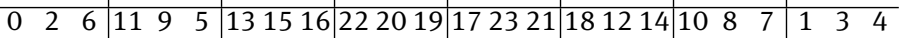

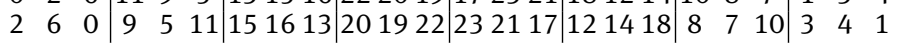

$$
\begin{aligned}
& \begin{array}{lll|lll|lll|lll|lll|lll|lll|lll}
6 & 0 & 2 & 5 & 11 & 9 & 16 & 13 & 15 & 19 & 22 & 20 & 21 & 17 & 23 & 14 & 18 & 12 & 7 & 10 & 8 & 4 & 1 & 3
\end{array}
\end{aligned}
$$

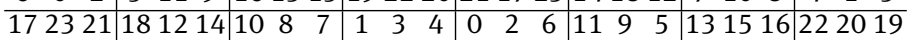

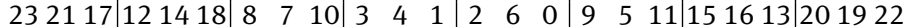

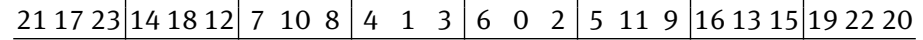

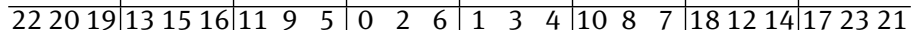

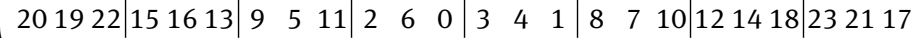

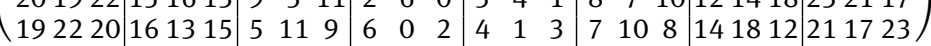

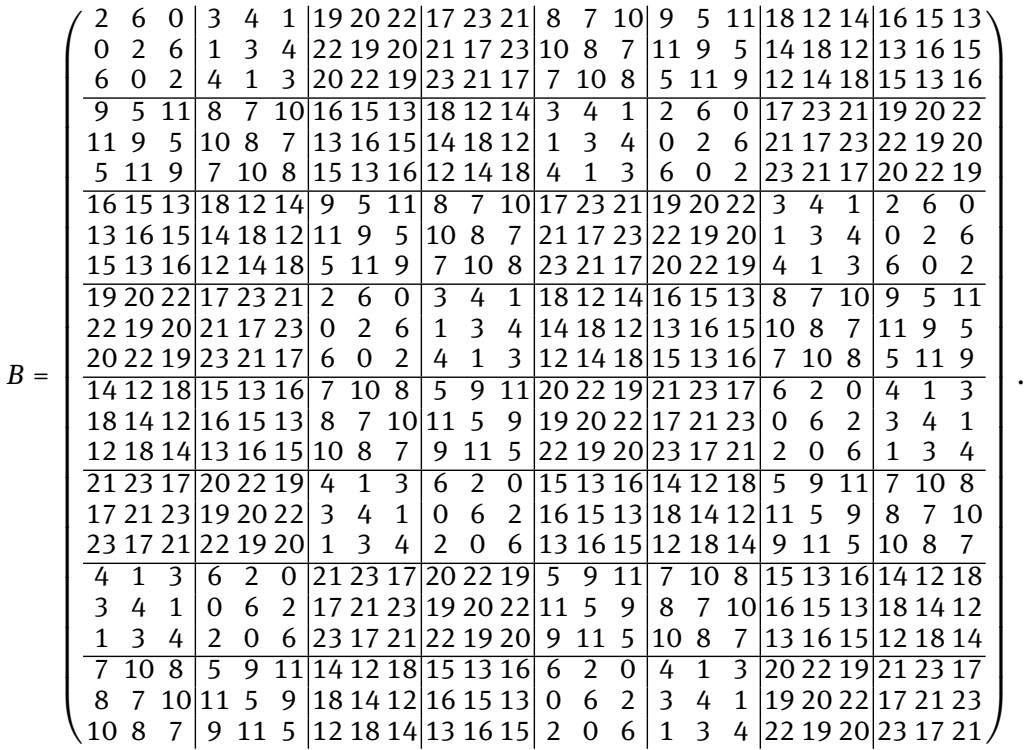

We introduce the following definition.

Definition 1. Let $L$ be a permutation of $0,1, \cdots, 4 n-1$. Denote $L=\left(L_{0}\left|L_{1}\right| L_{2} \mid L_{3}\right)$, where

$$
L_{i}=\left(l_{i}(0) l_{i}(1) \cdots l_{i}(n-1)\right), i=0,1,2,3 .
$$


$L$ is denoted by $S(4 n)$ if it satisfies the following 3 conditions.

$$
\begin{array}{ll}
\left\{l_{v}(j) \mid j \in I_{n}\right\}=\left\{4 n-1-l_{3-v}(j) \mid j \in I_{n}\right\}, & v=0,1, \\
l_{0}(j)+l_{2}(j)=l_{1}(j)+l_{3}(j), & j \in I_{n}, \\
l_{0}(j)+l_{2}(j)=l_{0}(n-1-j)+l_{2}(n-1-j), & j \in I_{n},
\end{array}
$$

Construction 2.4. If there is an $S(4 n)$ and there is a pair of strongly symmetrical OLS(n), then there is a pair of SPOLS(4n).

Proof We use Construction 2.2 by taking $m=4$. Let

$$
D=\left(\begin{array}{llll}
0 & 3 & 1 & 2 \\
2 & 1 & 3 & 0 \\
3 & 0 & 2 & 1 \\
1 & 2 & 0 & 3
\end{array}\right), \quad D^{\prime}=\left(\begin{array}{llll}
2 & 0 & 1 & 3 \\
1 & 3 & 2 & 0 \\
3 & 1 & 0 & 2 \\
0 & 2 & 3 & 1
\end{array}\right)
$$

Clearly, $\left(D, D^{\prime}\right)$ a pair of strongly symmetrical OLS(4). Let $\left(M, M^{\prime}\right)$ be a pair of strongly symmetrical OLS $(n)$.

Let $L=\left(L_{0}\left|L_{1}\right| L_{2} \mid L_{3}\right)$ be an S(4n). Denote

$$
L_{v}=\left(l_{v}(0) l_{v}(1) \cdots l_{v}(n-1)\right), v \in I_{4},
$$

For any $t \in I_{n}$, let

$$
\varphi_{u, v}(t)=\left\{\begin{array}{lr}
l_{v}(t), & u=0,2 \\
4 n-1-l_{3-v}(n-1-t), & u=1,3
\end{array}\right.
$$

and

$$
\varphi_{u, v}^{\prime}(t)=\varphi_{u, v}(t), \quad u, v \in I_{4} .
$$

Let

$$
T_{v}=\left\{l_{v}(j) \mid j \in I_{n}\right\}, v \in I_{4}, \quad T=\left\{T_{0}, T_{1}, T_{2}, T_{3}\right\} .
$$

Since $L$ is a permutation, $T$ is a partition of $I_{4 n}$. By (R1), $T_{v}=\left\{4 n-1-x \mid x \in T_{3-v}\right\}, v \in I_{4}$.

For fixed $v \in I_{4}$, if $t$ runs over $I_{n}$ then $\varphi_{0, v}(t), \varphi_{2, v}(t)$ run over $T_{v}$, and $\varphi_{1, v}(t), \varphi_{3, v}(t)$ runs over $\{4 n-1-$ $\left.x \mid x \in T_{3-v}\right\}$, which is exactly $T_{v}$. So, for any $u, v \in I_{4}, \varphi_{u, v}$ is a bijection from $I_{n}$ to $T_{v}$. By (2) $\varphi_{u, v}^{\prime}$ is also a bijection from $I_{n}$ to $T_{v}$.

Let $A, B$ be the matrices given by the Construction 2.2. We shall prove that $\varphi$ and $\varphi^{\prime}$ satisfy (L1)-(L6) in Construction 2.2. So, $(A, B)$ is a pair of SPOLS( $4 n)$ by Construction 2.2.

In fact, by (1), for any $t \in I_{n}$,

$$
\varphi_{0, v}(t)+\varphi_{3,3-v}(n-1-t)=l_{v}(t)+4 n-1-l_{v}(t)=4 n-1 .
$$

Similarly, one can prove that $\varphi_{1, v}(t)+\varphi_{2,3-v}(n-1-t)=4 n-1$. Thus (L1) and (L2) in Construction 2.2 are satisfied.

For any $t \in I_{n}$, by (1) and (R2) and (R3) it is readily verified the following.

$$
\begin{gathered}
\varphi_{0,0}(t)+\varphi_{0,2}(t)=\varphi_{0,0}(n-1-t)+\varphi_{0,2}(n-1-t), \\
\varphi_{0,1}(t)+\varphi_{0,3}(t)=\varphi_{0,0}(n-1-t)+\varphi_{0,2}(n-1-t), \\
\varphi_{0,1}(t)+\varphi_{0,3}(t)=\varphi_{0,1}(n-1-t)+\varphi_{0,3}(n-1-t) .
\end{gathered}
$$

By (1) we have

$$
\varphi_{0, v}(t)=4 n-1-\varphi_{1,3-v}(n-1-t)=\varphi_{2, v}(t)=4 n-1-\varphi_{3,3-v}(n-1-t) .
$$


By (3) we have

$$
\varphi_{0,0}(t)+\varphi_{1,1}(t)+\varphi_{2,2}(t)+\varphi_{3,3}(t)=2(4 n-1), t \in I_{n} .
$$

That is $\sum_{u \in I_{4}} \varphi_{u, d_{u, u}}(t)=2(4 n-1), t \in I_{n}$. Similarly, by (4) and (5) we have

$$
\sum_{u \in I_{4}} \varphi_{u, d_{u,\langle v+u\rangle_{4}}}(t)=2(4 n-1), v=1,2,3, t \in I_{n} .
$$

Thus for any $v \in I_{4}, t \in I_{n}, \varphi$ satisfies

$$
\sum_{u \in I_{4}} \varphi_{u, d_{u,\langle v+u\rangle_{4}}}(t)=2(4 n-1) .
$$

So,

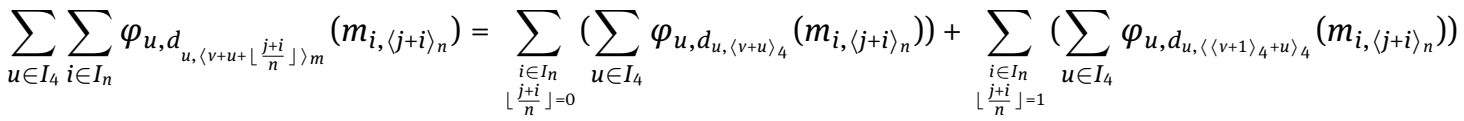

$$
\begin{aligned}
& =\sum_{\substack{i \in I_{n} \\
\left\lfloor\frac{j i}{n}\right\rfloor=0}} 2(4 n-1)+\sum_{\substack{i \in I_{n} \\
\left\lfloor\frac{j i}{n}\right\rfloor=1}} 2(4 n-1) \\
& =2 n(4 n-1) \text {. }
\end{aligned}
$$

It shows that the condition (L3) in Construction 2.2 is satisfied.

The condition (L4) in Construction 2.2 is satisfied by (3), (4) and (5). So (L5) and (L6) are satisfied by (2).

The following example is provided to explain Construction 2.4 and its proof.

Example 1. Let $\left(D, D^{\prime}\right)$ be the same as in Construction 2.4. Let $M=D, M^{\prime}=D^{\prime}$. Then $D, D^{\prime}, M, M^{\prime}$ are strongly symmetrical. Let

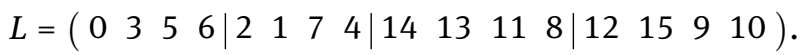

Clearly $L$ is an S(16). By Construction 2.4 we have

$$
A=\left(\begin{array}{cccc|cccc|cccc|cccc}
0 & 6 & 3 & 5 & 12 & 10 & 15 & 9 & 2 & 4 & 1 & 7 & 14 & 8 & 13 & 11 \\
5 & 3 & 6 & 0 & 9 & 15 & 10 & 12 & 7 & 1 & 4 & 2 & 11 & 13 & 8 & 14 \\
6 & 0 & 5 & 3 & 10 & 12 & 9 & 15 & 4 & 2 & 7 & 1 & 8 & 14 & 11 & 13 \\
3 & 5 & 0 & 6 & 15 & 9 & 12 & 10 & 1 & 7 & 2 & 4 & 13 & 11 & 14 & 8 \\
\hline 11 & 13 & 8 & 14 & 7 & 1 & 4 & 2 & 9 & 15 & 10 & 12 & 5 & 3 & 6 & 0 \\
14 & 8 & 13 & 11 & 2 & 4 & 1 & 7 & 12 & 10 & 15 & 9 & 0 & 6 & 3 & 5 \\
13 & 11 & 14 & 8 & 1 & 7 & 2 & 4 & 15 & 9 & 12 & 10 & 3 & 5 & 0 & 6 \\
8 & 14 & 11 & 13 & 4 & 2 & 7 & 1 & 10 & 12 & 9 & 15 & 6 & 0 & 5 & 3 \\
\hline 12 & 10 & 15 & 9 & 0 & 6 & 3 & 5 & 14 & 8 & 13 & 11 & 2 & 4 & 1 & 7 \\
9 & 15 & 10 & 12 & 5 & 3 & 6 & 0 & 11 & 13 & 8 & 14 & 7 & 1 & 4 & 2 \\
10 & 12 & 9 & 15 & 6 & 0 & 5 & 3 & 8 & 14 & 11 & 13 & 4 & 2 & 7 & 1 \\
15 & 9 & 12 & 10 & 3 & 5 & 0 & 6 & 13 & 11 & 14 & 8 & 1 & 7 & 2 & 4 \\
\hline 7 & 1 & 4 & 2 & 11 & 13 & 8 & 14 & 5 & 3 & 6 & 0 & 9 & 15 & 10 & 12 \\
2 & 4 & 1 & 7 & 14 & 8 & 13 & 11 & 0 & 6 & 3 & 5 & 12 & 10 & 15 & 9 \\
1 & 7 & 2 & 4 & 13 & 11 & 14 & 8 & 3 & 5 & 0 & 6 & 15 & 9 & 12 & 10 \\
4 & 2 & 7 & 1 & 8 & 14 & 11 & 13 & 6 & 0 & 5 & 3 & 10 & 12 & 9 & 15
\end{array}\right), B=\left(\begin{array}{ccccc|cccc|cccc|cccc}
11 & 14 & 13 & 8 & 5 & 0 & 3 & 6 & 7 & 2 & 1 & 4 & 9 & 12 & 15 & 10 \\
13 & 8 & 11 & 14 & 3 & 6 & 5 & 0 & 1 & 4 & 7 & 2 & 15 & 10 & 9 & 12 \\
8 & 13 & 14 & 11 & 6 & 3 & 0 & 5 & 4 & 1 & 2 & 7 & 10 & 15 & 12 & 9 \\
14 & 11 & 8 & 13 & 0 & 5 & 6 & 3 & 2 & 7 & 4 & 1 & 12 & 9 & 10 & 15 \\
\hline 2 & 7 & 4 & 1 & 12 & 9 & 10 & 15 & 14 & 11 & 8 & 13 & 0 & 5 & 6 & 3 \\
4 & 1 & 2 & 7 & 10 & 15 & 12 & 9 & 8 & 13 & 14 & 11 & 6 & 3 & 0 & 5 \\
1 & 4 & 7 & 2 & 15 & 10 & 9 & 12 & 13 & 8 & 11 & 14 & 3 & 6 & 5 & 0 & 0 \\
7 & 2 & 1 & 4 & 9 & 12 & 15 & 10 & 11 & 14 & 13 & 8 & 5 & 0 & 3 & 6 \\
\hline 9 & 12 & 15 & 10 & 7 & 2 & 1 & 4 & 5 & 0 & 3 & 6 & 11 & 14 & 13 & 8 \\
15 & 10 & 9 & 12 & 1 & 4 & 7 & 2 & 3 & 6 & 5 & 0 & 13 & 8 & 11 & 14 \\
10 & 15 & 12 & 9 & 4 & 1 & 2 & 7 & 6 & 3 & 0 & 5 & 8 & 13 & 14 & 11 \\
12 & 9 & 10 & 15 & 2 & 7 & 4 & 1 & 0 & 5 & 6 & 3 & 14 & 11 & 8 & 13 \\
\hline 0 & 5 & 6 & 3 & 14 & 11 & 8 & 13 & 12 & 9 & 10 & 15 & 2 & 7 & 4 & 1 \\
6 & 3 & 0 & 5 & 8 & 13 & 14 & 11 & 10 & 15 & 12 & 9 & 4 & 1 & 2 & 7 \\
3 & 6 & 5 & 0 & 13 & 8 & 11 & 14 & 15 & 10 & 9 & 12 & 1 & 4 & 7 & 2 \\
5 & 0 & 3 & 6 & 11 & 14 & 13 & 8 & 9 & 12 & 15 & 10 & 7 & 2 & 1 & 4
\end{array}\right) .
$$

It is readily verified that $(A, B)$ is a pair of SPOLS(16). 
Definition 2. Let $H$ be an $m \times n$ matrix over $I_{m n}$ consisting of distinct numbers. $H$ is called a row magic rectangle if

$$
\sum_{j=0}^{n-1} h_{i, j}=\frac{n(m n-1)}{2}, i \in I_{m}
$$

A row magic rectangle $H$ is called centre-complementary, denoted by $(m, n)$-CCRMR, if $H$ satisfies

$$
h_{i, j}+h_{m-1-i, n-1-j}=m n-1, i \in I_{m}, j \in I_{n} .
$$

See similar definition in [14].

Construction 2.5. Let $n \equiv 3(\bmod 6), n \neq 3$, if there is a $\left(3, \frac{n}{3}\right)-C C R M R$, then there is a pair of SPOLS $(n)$.

Proof Let $H$ be a $\left(3, \frac{n}{3}\right)$-CCRMR over $I_{n}$. Define a penmutation $\sigma$ of $I_{n}$ :

$$
\sigma(i)=h_{\langle i\rangle_{3},\left\lfloor\frac{i}{3}\right\rfloor}, \quad i \in I_{n} .
$$

Let $U, V$ be matrices of order $n$ over $I_{n}$, where

$$
u_{i, j}=\sigma\left(\langle 2 i+j+1\rangle_{n}\right), \quad v_{i, j}=\sigma\left(\langle 2 i-j\rangle_{n}\right), \quad i, j \in I_{n} .
$$

It is readily verified that $(U, V)$ is a pair of $\operatorname{OLS}(n)$. We shall prove that $U$ and $V$ is a pair of SPOLS $(n)$.

First we shall prove that $U, V$ are weakly pandiagonal. Clearly, the left pandiagonals of $U$ and the right pandiagonals of $V$ are transversals. For the left pandiagonal of $U$, fixed $w \in I_{n}$, let $w=3 s+t, s \in I_{\frac{n}{3}}, t \in I_{3}$. By (6), (8) and (9) we have

$$
\sum_{i=0}^{n-1} u_{i,\langle i+w\rangle_{n}}=\sum_{i=0}^{n-1} \sigma\left(\left\langle 2 i+\langle i+w\rangle_{n}+1\right\rangle_{n}\right)=\sum_{i=0}^{n-1} \sigma\left(\langle 3(i+s)+t+1\rangle_{n}\right),
$$

We have $\left\{\langle 3(i+s)+t+1\rangle_{n} \mid i \in I_{n}\right\}=3\left\{3 i+\langle t+1\rangle_{3} \mid i \in I_{\frac{n}{3}}\right\}$. In fact, since $t+1=3\left\lfloor\frac{t+1}{3}\right\rfloor+\langle t+1\rangle_{3}$ and there is an integer $q$ such that $i+s+\left\lfloor\frac{t+1}{3}\right\rfloor=q \cdot \frac{n}{3}+\left\langle i+s+\left\lfloor\frac{t+1}{3}\right\rfloor\right\rangle_{\frac{n}{3}}$, we have

$$
\begin{aligned}
\left\{\langle 3(i+s)+t+1\rangle_{n} \mid i \in I_{n}\right\}=\{\langle 3(i+ & \left.\left.\left.s+\left\lfloor\frac{t+1}{3}\right\rfloor\right)+\langle t+1\rangle_{3}\right\rangle_{n} \mid i \in I_{n}\right\} \\
& =\left\{\left\langle 3\left\langle i+s+\left\lfloor\frac{t+1}{3}\right\rfloor\right\rangle_{\frac{n}{3}}+\langle t+1\rangle_{3}\right\rangle_{n} \mid i \in I_{n}\right\} \\
& =\left\{3\left\langle i+s+\left\lfloor\frac{t+1}{3}\right\rfloor\right\rangle_{\frac{n}{3}}+\langle t+1\rangle_{3} \mid i \in I_{n}\right\} \\
& =3\left\{3 i+\langle t+1\rangle_{3} \mid i \in I_{\frac{n}{3}}\right\}
\end{aligned}
$$

Thus the equality (10) is $3 \sum_{i=0}^{\frac{n}{3}-1} \sigma\left(3 i+\langle t+1\rangle_{3}\right)$. It also equals to $3 \sum_{i=0}^{\frac{n}{3}-1} h_{\langle t+1\rangle_{3}, i}$ by the equality (8). Further, it is just $\frac{n(n-1)}{2}$ by (6). So the sum of the $n$ numbers of each right pandiagonal of $U$ is the same. Similarly, the sum of the $n$ numbers of each left pandiagonal of $V$ is the same. Thus $U, V$ are weakly pandiagonal.

Now we prove that $U, V$ are strongly symmetrical. For any $i \in I_{n}$, let $q=\left\lfloor\frac{i}{3}\right\rfloor, r=\langle i\rangle_{3}$, then $i=3 q+r$, $q \in I_{\frac{n}{3}}, r \in I_{3}$. So we have $n-1-i=3\left(\frac{n}{3}-1-q\right)+2-r$. Thus by (7) and (8) we have

$$
\sigma(i)+\sigma(n-1-i)=h_{r, q}+h_{2-r, \frac{n}{3}-1-q}=n-1 \text {. }
$$

for $i, j \in I_{n}$, we have

$$
u_{n-1-i, n-1-j}=\sigma\left(\langle 2(n-1-i)+(n-1-j)+1\rangle_{n}\right)=\sigma\left(\left\langle n-1-\langle 2 i+j+1\rangle_{n}\right\rangle_{n}\right),
$$

which is just $\sigma\left(n-1-\langle 2 i+j+1\rangle_{n}\right)$. So,

$$
u_{i, j}+u_{n-1-i, n-1-j}=\sigma\left(\langle 2 i+j+1\rangle_{n}\right)+\sigma\left(n-1-\langle 2 i+j+1\rangle_{n}\right)=n-1 .
$$

Thus $U$ is strongly symmetrical. Similarly, one can prove that $V$ is strongly symmetrical. Therefore, $(U, V)$ is a pair of $\operatorname{SPOSL}(n)$. 
An example is provided to explain the construction 2.5 .

Example 2. Let $n=9$, and

$$
H=\left(\begin{array}{lll}
0 & 7 & 5 \\
6 & 4 & 2 \\
3 & 1 & 8
\end{array}\right)
$$

Clearly, $H$ is a $(3,3)$-CCRMR. By Construction 2.5, define a permutation of $\sigma$ over $I_{9}$ :

$$
\sigma(i)=h_{\langle i\rangle_{3},\left\lfloor\frac{i}{3}\right\rfloor}, \quad i \in I_{9} .
$$

That is

$$
\sigma=\left(\begin{array}{lllllllll}
0 & 1 & 2 & 3 & 4 & 5 & 6 & 7 & 8 \\
0 & 6 & 3 & 7 & 4 & 1 & 5 & 2 & 8
\end{array}\right)
$$

Let $(U, V)$ be a pair of OLS(9) given by (9), where

$$
u_{i, j}=\sigma\left(\langle 2 i+j+1\rangle_{9}\right), \quad v_{i, j}=\sigma\left(\langle 2 i-j\rangle_{9}\right), \quad i, j \in I_{9}
$$

So,

$$
U=\left(\begin{array}{lllllllll}
6 & 3 & 7 & 4 & 1 & 5 & 2 & 8 & 0 \\
7 & 4 & 1 & 5 & 2 & 8 & 0 & 6 & 3 \\
1 & 5 & 2 & 8 & 0 & 6 & 3 & 7 & 4 \\
2 & 8 & 0 & 6 & 3 & 7 & 4 & 1 & 5 \\
0 & 6 & 3 & 7 & 4 & 1 & 5 & 2 & 8 \\
3 & 7 & 4 & 1 & 5 & 2 & 8 & 0 & 6 \\
4 & 1 & 5 & 2 & 8 & 0 & 6 & 3 & 7 \\
5 & 2 & 8 & 0 & 6 & 3 & 7 & 4 & 1 \\
8 & 0 & 6 & 3 & 7 & 4 & 1 & 5 & 2
\end{array}\right), V=\left(\begin{array}{lllllllll}
0 & 8 & 2 & 5 & 1 & 4 & 7 & 3 & 6 \\
3 & 6 & 0 & 8 & 2 & 5 & 1 & 4 & 7 \\
4 & 7 & 3 & 6 & 0 & 8 & 2 & 5 & 1 \\
5 & 1 & 4 & 7 & 3 & 6 & 0 & 8 & 2 \\
8 & 2 & 5 & 1 & 4 & 7 & 3 & 6 & 0 \\
6 & 0 & 8 & 2 & 5 & 1 & 4 & 7 & 3 \\
7 & 3 & 6 & 0 & 8 & 2 & 5 & 1 & 4 \\
1 & 4 & 7 & 3 & 6 & 0 & 8 & 2 & 5 \\
2 & 5 & 1 & 4 & 7 & 3 & 6 & 0 & 8
\end{array}\right),
$$

It is readily verified that $(U, V)$ is a pair of $\operatorname{SPOLS}(9)$.

\section{Proof of Theorem 1.3}

In this section we consider the exitence of a pair of SPOLS $(n)$. There doesn't exist a pair of SPOLS $(n)$ if $n \equiv 2(\bmod 4)$ by Lemma 1.1. To prove Theorem 1.3 it remains to check the following three cases: $(1) n \equiv 1,5$ $(\bmod 6) ;(2) n \equiv 3(\bmod 6) ;(3) n \equiv 0(\bmod 4)$.

Case 1. $n \equiv 1,5(\bmod 6)$

Lemma 3.1. There exists a pair of $S P O L S(n)$ if $n \equiv 1,5(\bmod 6)$.

Proof Let $U=\left(u_{i, j}\right)_{n \times n}, V=\left(v_{i, j}\right)_{n \times n}$, where

$$
u_{i, j}=\langle 2 i+j+1\rangle_{n}, \quad v_{i, j}=\langle 2 i-j\rangle_{n}, \quad i, j \in I_{n}
$$

$(U, V)$ is a pair of OLS $(n)$ over $I_{n}$. Now we prove that $U, V$ are strongly symmetrical and weakly pandiagonal. It is enough to prove $U$ is strongly symmetrical and weakly pandiagonal. Similarly we have $V$.

For any $i, j \in I_{n}$, we have

$$
\begin{aligned}
u_{i, j}+u_{n-1-i, n-1-j} & =\langle 2 i+j+1\rangle_{n}+\langle 2(n-1-i)+(n-1-j)+1\rangle_{n} \\
& =\langle 2 i+j+1\rangle_{n}+\langle 3 n-1-(2 i+j+1)\rangle_{n} \\
& =\langle 2 i+j+1\rangle_{n}+\left\langle n-1-\langle 2 i+j+1\rangle_{n}\right\rangle_{n} \\
& =n-1 .
\end{aligned}
$$


So $U$ is strongly symmetrical.

We consider the pandiagonals of $U$. For any $k \in I_{n}$, $\operatorname{since} \operatorname{gcd}(3, n)=1$, we have

$$
\begin{aligned}
\left\{u_{i,\langle i+k\rangle_{n}} \mid i \in I_{n}\right\} & =\left\{\left\langle 2 i+\langle i+k\rangle_{n}+1\right\rangle_{n} \mid i \in I_{n}\right\} \\
& =\left\{\langle 3 i+k+1\rangle_{n} \mid i \in I_{n}\right\} \\
& =\left\{\langle i+k+1\rangle_{n} \mid i \in I_{n}\right\} \\
& =I_{n} .
\end{aligned}
$$

It shows that each right pandiagonal of $U$ is a transversal. Similarly, each left pandiagonal of $U$ is also a transversal. Thus $U$ is weakly pandiagonal.

Case 2. $n \equiv 3(\bmod 6)$

Lemma 3.2. There is $a(3, n)$-CCRMR for odd $n \geq 3$.

Proof Let $n$ be odd, $n \geq 3$, denote $n=4 s+w, w \in\{3,5\}, s \geq 0$. We perform induction on $s$. If $s=0$, a $(3,3)$-CCRMR is given by Example 2, and a $(3,5)$-CCRMR is given below.

$$
\left(\begin{array}{ccccc}
0 & 13 & 6 & 12 & 4 \\
3 & 9 & 7 & 5 & 11 \\
10 & 2 & 8 & 1 & 14
\end{array}\right)
$$

For $s \geq 0$, supppose that $A(s, w)$ is a $(3,4 s+w)$-CCRMR. Then the row sum of $A(s, w)$ is

$$
(4 s+w)(12 s+3 w-1) / 2 .
$$

The sum of each pair of symmetrical elements is $12 s+3 w-1$. Let

$$
L=\left(\begin{array}{ll}
0 & 5 \\
1 & 4 \\
2 & 3
\end{array}\right), R(s, w)=\left(\begin{array}{lc}
12 s+3 w+8 & 12 s+3 w+9 \\
12 s+3 w+7 & 12 s+3 w+10 \\
12 s+3 w+6 & 12 s+3 w+11
\end{array}\right) .
$$

Denote

$$
A(s+1, w)=\left(L\left|A(s, w)+6 J_{3 \times(4 s+w)}\right| R(s, w)\right) .
$$

It is readily verified that the elements of $A(s+1, w)$ run over $I_{n+12}$. By calculation, for any $i \in I_{3}$, the sum of the elements of the $i$-th row of $A(s+1, w)$ is

$$
\frac{n(3 n-1)}{2}+6 n+2(3 n)+22=\frac{(4(s+1)+w)(12(s+1)+3 w-1)}{2},
$$

which is independent on $i$. The sum of each pair of symmetrical elements of $A(s+1, w)$ is $12 s+3 w+11=$ $12(s+1)+3 w-1$, which is also independent on $i$. Thus $A(s+1, w)$ is a $(3,4(s+1)+w)$-CCRMR. There is a $(3, n)$-CCRMR for any odd $n \geq 3$ by induction.

Lemma 3.3. There exists a pair of $\operatorname{SPOLS}(n)$ if $n \equiv 3(\bmod 6)$ and $n \neq 3$.

Proof It follows by Construction 2.5 and Lemma 3.2.

Theorem 3.4. There is a pair of SPOLS( $n)$ for any odd $n \geq 5$.

Proof It follows by Lemma 3.1 and Lemma 3.3.

Case 3. $n \equiv 0(\bmod 4)$

Let $n=4 k, k \geq 1$. We should deal with the case $k \equiv 2(\bmod 4)$ and the case $k \equiv 0,1,3(\bmod 4)$.

Lemma 3.5. There is a pair of SPOLS(4k) for any positive integer $k \equiv 2(\bmod 4)$. 
Proof The $\left(D, D^{\prime}\right)$ given in Section 1 is a pair of SPOLS(8). Lemma 2.3 gives a pair of SPOLS(24). Thus there is a pair of $\operatorname{SPOLS}(4 k), k=2,6$. For $k \equiv 2(\bmod 4), k \geq 10$, there is a pair of $\operatorname{SPOLS}\left(\frac{k}{2}\right)$ by Theorem 3.4. Since there is a pair of SPOLS(8), there is a pair of SPOLS( $4 k)$ by Construction 2.1.

Now we consider the case $k \equiv 0,1,3(\bmod 4)$.

Lemma 3.6. There is an $S(4 k)$ if $k \equiv 0,1,3(\bmod 4)$ and $k \geq 4$, .

Proof There is an S( $4 k)$ for $k=4,5,7$ as follows.

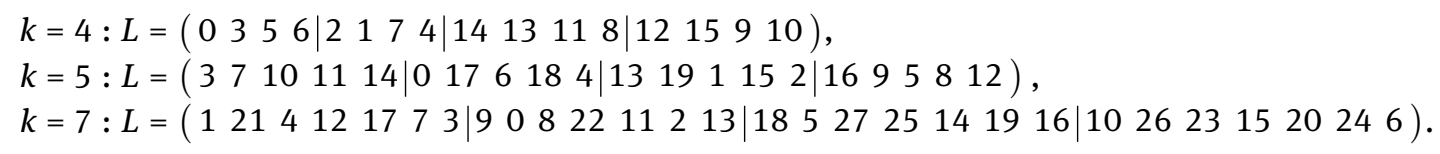

Suppose that there is an $\mathrm{S}(4 k)$ for any $k \geq 4, k \equiv 0,1,3(\bmod 4)$,

$$
L=\left(L_{0} L_{1} L_{2} L_{3}\right)
$$

where the $j$-position of $L_{i}$ is $l_{i}(j), i \in I_{4}, j \in I_{k}$. Let

$$
H=\left(H_{0} H_{1} H_{2} H_{3}\right)
$$

be the $\mathrm{S}(16)$ given above. We shall construct an $\mathrm{S}(4(k+4))$. Let

$$
\widetilde{L}=\left(\widetilde{L}_{0} \widetilde{L}_{1} \widetilde{L}_{2} \widetilde{L}_{3}\right)
$$

where

$$
\begin{aligned}
& \widetilde{L}_{0}=\left(h_{0}(0), h_{0}(1), l_{0}(0)+8, \cdots, l_{0}(k-1)+8, h_{0}(2), h_{0}(3)\right), \\
& \widetilde{L}_{1}=\left(h_{1}(0), h_{1}(1), l_{1}(0)+8, \cdots, l_{1}(k-1)+8, h_{1}(2), h_{1}(3)\right), \\
& \widetilde{L}_{2}=\left(h_{2}(0)+16, h_{2}(1)+16, l_{2}(0)+8, \cdots, l_{2}(k-1)+8, h_{2}(2)+16, h_{2}(3)+16\right), \\
& \widetilde{L}_{3}=\left(h_{3}(0)+16, h_{3}(1)+16, l_{3}(0)+8, \cdots, l_{3}(k-1)+8, h_{3}(2)+16, h_{3}(3)+16\right) .
\end{aligned}
$$

Clearly, the elements of $\widetilde{L}$ run over $I_{4(k+4)}$, and it satisfy the conditions (R1)-(R3). Thus $\widetilde{L}$ is an S(4(k+4)). The proof is completed by induction.

Example 3. By using the $\mathrm{S}(16)$ given in Lemma 3.6 we can get an $\mathrm{S}(32)$ as follows:

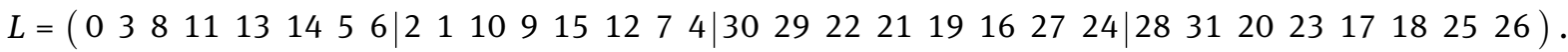

Lemma 3.7. There is a pair of SPOLS(4k) for $k \equiv 0,1,3(\bmod 4)$ and $k>4$.

Proof Let $k \equiv 0,1,3(\bmod 4), k>4$. There is a pair of strongly symmetrical OLS $(k)$ by Lemma 1.1, and there is an $\mathrm{S}(4 k)$ by Lemma 3.6. Thus there is a pair of SPOLS( $4 k)$ by Construction 2.4.

Theorem 3.8. There is a pair of SPOLS(4k) for $k \geq 2$ and $k \neq 3$.

Proof The result follows from Lemma 3.5 and Lemma 3.7.

Lemma 3.9. There is no $\operatorname{SPOLS}(4)$.

Proof Suppose that there is a pair of SPOLS(4) over $I_{4},(A, B)$. Let

$$
U=4 A+B=\left(\begin{array}{llll}
a_{1} & b_{1} & a_{2} & b_{2} \\
c_{1} & d_{1} & c_{2} & d_{2} \\
a_{3} & b_{3} & a_{4} & b_{4} \\
c_{3} & d_{3} & c_{4} & d_{4}
\end{array}\right)
$$


Let

$$
\begin{array}{ll}
e=a_{1}+a_{2}+a_{3}+a_{4}, & f=b_{1}+b_{2}+b_{3}+b_{4}, \\
g=c_{1}+c_{2}+c_{3}+c_{4}, & h=d_{1}+d_{2}+d_{3}+d_{4} .
\end{array}
$$

Then $e+f=e+g=60$, hence $f=g$. Since $A$ and $B$ are strongly symmetrical, we have $b_{1}+c_{4}=c_{1}+b_{4}=$ $b_{2}+c_{3}=c_{2}+b_{3}=15$. Thus $f+g=60$. So, $f=g=30$. On the other hand, since $A$ is weakly pandiagonal, $b_{2}+c_{2}+b_{3}+c_{3}=b_{2}+c_{1}+b_{3}+c_{4}=30$. So $c_{2}+c_{3}=c_{1}+c_{4}, c_{1}+c_{2}+c_{3}+c_{4}=2\left(c_{1}+c_{4}\right)=30$, it follows that $c_{1}+c_{4}=15$. Previously we have proved that $c_{1}+b_{4}=15$, so $c_{4}=b_{4}$, a contradiction. Thus there is no SPOLS(4).

Proof of Theorem1.3 The proof follows by Combining Theorem 3.4, Theorem 3.8 and Lemma 3.9.

Acknowledgement: The authors would like to thank Professor L. Zhu of Suzhou University for his encouragement. Thank Professor Lei Jianguo of Hebei Normal University. Thank anonymous reviewers for careful reading and helpful suggestions.

Research supported by the Natural Science Foundations of China (No.11871417). The first author is also supported by Program for the Top Young Innovative Talents of Yancheng Teachers University.

\section{References}

[1] R. C. Bose, S. S. Shrikhande, E. T. Parker, Further results on the construction of mutually orthogonal Latin squares and the falsity of Euler's Conjecture, Canad. J. Math., 12(1960): 189-203.

[2] J. W. Brown, F. Cherry, L. Most, M. Most, E. T. Parker, W. D. Wallis. The spectrum of orthogonal diagonal latin squares, in Graphs, Matrices and Designs, R. S. Rees, ed. Dekker, New York, 1993, 43-49.

[3] H. Cao, W. Li, Existence of strong symmetric self-orthogonal diagonal Latin squares, Discrete Math., 311(2011): 841-843.

[4] K. Wang, K. Chen, A short disproof of Euler's conjecture based on quasi-difference matrices and difference matrices, Discrete Math., 341(4)(2018): 1114-1119.

[5] C. J. Colbourn, J. H. Dinitz (eds), Handbook of Combinatorial Designs, 2nd Edition. Chapman and Hall/CRC. Boca Raton FL, 2007.

[6] K. J. Danhof, N. C. K. Phillips, W. D. Wallis, On self-orthogonal diagonal Latin squares, J. Combin. Math. Combin. Comput., 8(1990): 3-8.

[7] J. Denes, A. D. Keedwell, Latin squares and their applications, Academic Press Inc, 1974.

[8] B. Du, H. Cao, Existence of strongly symmetric self-orthogonal diagonal latin squares, Acta Math. Appl. Sin. (Chinese), 25(2002): 187-189.

[9] L. Euler, Recherches sur une nouvelle espece de quarres magiques, Verh Genootsch der Wet Vlissingen, 9(1782): 85-232.

[10] K. Heinrich, A. J. W. Hilton, Doubly diagonal orthogonal Latin squares, Discrete Math., 46(1983): 173-182.

[11] W. D. Wallis, L. Zhu, Existences of orthogonal diagonal latin squares, Ars Combin., 12(1981): 51-68.

[12] C. Xu, Z. Lu, Pandiagonal magic squares, Lecture Notes in Computer Science, (959)1995: 388-391.

[13] Y. Zhang, W. Li, J. Lei, Orthogonal pandiagonal Latin squares, Acta Math. Sin. (Engl. Ser.), 6(2013): 1089-1094.

[14] C. Zhou, W. Li, Y. Zhang, R. Su, Existence of magic 3-dimensional rectangles, J. Combin. Des. 26(6) (2018) 280-309.

[15] L. Zhu, A short disproof of Euler's conjecture concerning orthogonal Latin squares, Ars Combin., 1982: 47-55. 